\title{
Location Aware MobiFeed System for Mobile Users
}

\author{
Jadhav Komal K ${ }^{1}$, Prof. Mulik AT ${ }^{2}$ \\ Student, Computer Science \& Engg, DACOE, Karad, India ${ }^{1}$ \\ Professor, Computer Science \& Engg, DACOE, Karad, India ${ }^{2}$
}

\begin{abstract}
A location aware MobiFeed system enables convenient customers to share geo-marked customer delivered messages, e.g., a customer can get nearby messages that are the most noteworthy to her. Existing LANF structures simply send the most appropriate geo-marked messages to their customers. Shockingly, the genuine confinement of such a present approach is, to the point that, a news reinforce may contain messages related to a comparable territory (i.e., reason for interest) or a comparative order of regions (e.g., food, incitement or wear). We battle that varying qualities is a basic component for zone careful news sustains in light of the way that it helps customers find new places additionally, exercises. In this paper we propose MobiFeed framework which permit the client to choose the classification and in view of that classification client can get news of his/her decision.
\end{abstract}

Keywords: Location Aware, MobiFeed, Location Aware News Feed.

\section{INTRODUCTION}

An area mindful news sustain framework empowers portable clients to share geo-labelled client produced messages, e.g., a client can get close-by messages that are the most applicable to her. In this paper, we display MobiFeed that is a system intended for booking news nourishes for portable clients. MobiFeed comprises of three key capacities, area expectation, significance measure, and news nourish scheduler.

The area forecast capacity is intended to foresee a versatile client's areas in light of a current way expectation calculation. The pertinence measure capacity is actualized by joining the vector space demonstrate with non-spatial and spatial variables to decide the importance of a message to a client. The news sustain scheduler works with the other two capacities to produce news bolsters for a versatile client at her current and anticipated areas with the best general quality. To guarantee that MobiFeed can scale up to a bigger number of messages, we outline heuristic news sustain scheduler.

A news encourage is a typical usefulness of existing area mindful informal organization frameworks. It empowers portable clients to post geo-labeled messages and get close-by client created messages, e.g.," Alice can get 4 messages that are the most important to her among the messages inside $1 \mathrm{~km}$ from her area at regular intervals". Since an area mindful interpersonal organization framework as a rule has an enormous number of messages, there are many messages in a questioning client's region. Combined with client portability, a key test for the area mindful news encourage framework is the way to effectively plan the $\mathrm{k}$ most significant messages for a client and show them on the client's cell phone.

In spite of the fact that area mindful news bolster and interpersonal organization frameworks have pulled in a considerable measure of consideration from various research groups, none of these applications has concentrated on the most proficient method to calendar news bolsters for portable clients. As opposed to GeoFeed MobiFeed concentrates on difficulties in giving area mindful news nourishes to versatile clients. We outline an area mindful MobiFeed scheduler that works with our area expectation and message importance measure capacities to give newsfeeds to portable clients. In this paper, we exhibit MobiFeed system intended for informal community frameworks to calendar news nourishes for portable clients.

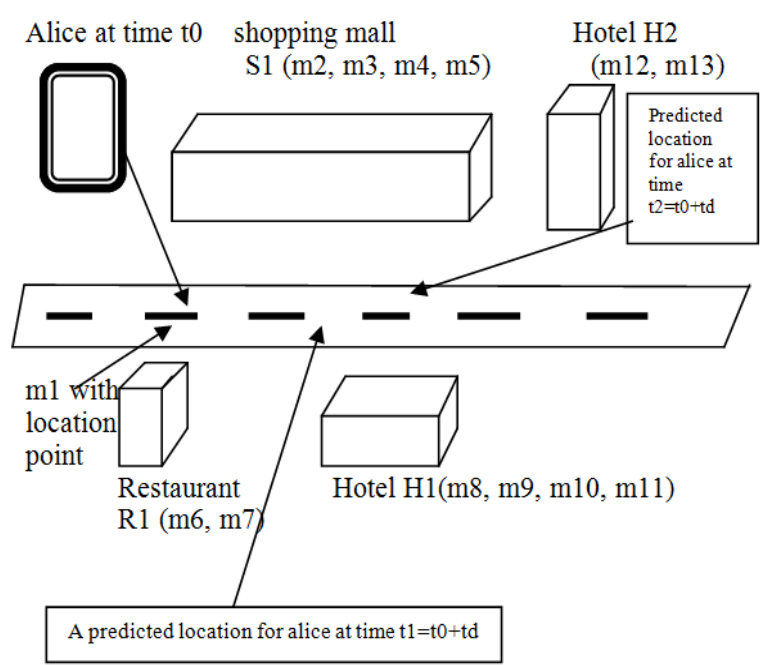

Fig.1. Location aware news Scheduling

Fig.1. portrays an application situation or a scene as its geo-area. Alice can likewise issue Location aware 
IARJSET

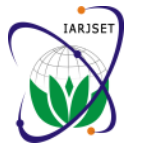

International Advanced Research Journal in Science, Engineering and Technology

National Conference on Innovative Applications and Research in Computer Science and Engineering (NCIARCSE-2017)

AGTI's Dr. Daulatrao Aher College Engineering, Vidyanagar Extension, Karad

Vol. 4, Special Issue 4, January 2017

MobiFeed inquiry to recover the $\mathrm{k}$ most important messages inside her predefined extend separate D from her area. MobiFeed comprises of three key capacities: area expectation, pertinence measure, and news encourage scheduler. Given a client users area users location at the present time $\mathrm{t} 0$, users required least message show time $\mathrm{td}$, users predefined extend remove $\mathrm{D}$, users asked for number of messages per news nourish, and a look-ahead strides and, the area forecast work gauges $\mathrm{n}$ future areas for $\mathrm{u}$ at timest $1=\mathrm{t} 0+\mathrm{td}, \mathrm{t} 2=\mathrm{t} 0+2 \times \mathrm{td}, \ldots$, and $\mathrm{tn}=\mathrm{t} 0+\mathrm{n} \times \mathrm{td}$, the pertinence measure work ascertains the significance score of every applicant message with a geo-area meeting anyuser's question district and the news encourage scheduler produces news bolsters from the competitor messages for user's inquiry areas at $\mathrm{t} 0, \mathrm{t} 1, \ldots .$. , th with the best aggregate significance score. Planning a versatile and compelling MobiFeed scheduler has a few key difficulties. A message has a lifetime concerning a client's development. A message can be a hopeful message for a few sequential or non-back to back news bolsters. The base show eras of these news encourages constitute the message's lifetime. The scheduler ought to choose at most $\mathrm{k}$ applicant messages for news encourage inside their lifetime interims to such an extent that the general nature of a client's news nourishes is augmented.

The pertinence of a message to a client is exceedingly changing. Since we consider the separation between a message and a client as one of the variables in the pertinence measure, the importance of a message could change for a client at various areas. A client wants to have the most applicable message at the highest point of an outcome list. The pertinence of a message showed on a screen ought to be weighted by its position. For instance, the most elevated weight is given to the message showed at the top on the screen. The online scheduler ought to be effective with the end goal that it could scale up to countless.

The commitments of this paper are condensed as takes after:

- We plan the area expectation work in light of the way forecast calculation and consolidate the vector space show with spatial and non-spatial variables to characterize the pertinence measure work.

- We consolidate area expectation to the procedure of area mindful news sustain era, subsequently detailing a novel n-look ahead news bolster planning structure to enhance the general nature of numerous news encourages for moving clients.

- We exhibit an ideal scheduler by finding the most extreme weight coordinating in a weighted bipartite diagram; we likewise give accuracy verification and multifaceted nature examination for our ideal scheduler.

- We display the choice issue as a most extreme stream issue to locate the base aggregate differing qualities of an arrangement of $n+1$ news bolsters for a client in view of the client determined differences limitation.
- We propose a three-arrange heuristic way to deal with take care of the improvement issue. The main stage takes care of a base cost stream issue to ensure the base aggregate assorted qualities in an arrangement of $n+1$ news sustains. The second stage delivers a recharge up-to$\mathrm{k}$ issue to augment the aggregate importance of these news encourages.

In this location aware mobi sustain framework system we have used the key modules:

1. Client Module.

2. Server Module.

3. Web Search Module.

1) Client Module- The differences rule is proposed for security safeguarding information distributing. Essentially, this guideline is utilized to sum up non-delicate in a class of records with the end goal that the touchy quality accomplishes the differing qualities requirement, to ensure the security of distributed information. In this work, we concentrate on an alternate issue since intends to amplify the significance of news encourages for portable clients while news nourishes fulfill the differing qualities imperative. Clients can impart their areas to companions without uncovering any area data to the informal community framework or other unapproved clients.

2) Server Module- Most existing news encourage frameworks just give distribute/subscribe administrations that basically forward messages to subscribed clients. Area mindfulness into news sustain framework, which empowers a message to be connected with a spatial degree to control where clients can get it. We proposed a structure that is intended to timetable news nourishes for portable clients. A few frameworks empower portable clients to communicate or get open geo-labeled messages, however they don't consider any non-spatial perspectives (e.g., client inclinations) or timetable messages for versatile clients in light of their movements. Mobi-Feed separates itself from all current news nourish frameworks in that it empowers clients to post message with spatial degree as opposed to static point areas, and considers their areas when processing news encourage for them. Mobi-Feed minimizes the framework overhead to deliver the area mindful news sustain, and ensures a specific reaction time for every client to acquire the asked for area mindful news bolster. Mobi-Feed additionally bolsters area mindful news sustain work for its versatile clients. Trial comes about, in light of genuine and manufactured information, demonstrate that Mobi-Feed outflanks existing news sustain frameworks regarding reaction time and support cost.

3) Web Search Module- The procedure of web inquiry frameworks varies from that of recommender frameworks since it includes an express client question (i.e., watchwords). The inquiry, in any case, is additionally vague and has more than one elucidation. One conceivable approach to deliver this issue is to create an arrangement 
IARJSET

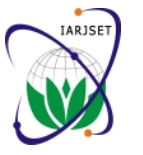

International Advanced Research Journal in Science, Engineering and Technology

National Conference on Innovative Applications and Research in Computer Science and Engineering (NCIARCSE-2017)

AGTI's Dr. Daulatrao Aher College Engineering, Vidyanagar Extension, Karad

Vol. 4, Special Issue 4, January 2017

of enhanced outcomes that cover diverse understandings of the objective inquiry. In particular, the item broadening approaches in the writing can be delegated either certain or express. Verifiable methodologies, accept that comparable records will cover comparative parts of an inquiry. Their essential thought is to iteratively choose.

\section{RELATED WORK}

A) Geo-Feed: A Location-Aware News Feed System: An area mindful news encourage framework that considers the spatial degrees of messages and client areas when settling on the chose news bolster. Geo-Feed is furnished with three diverse methodologies, in particular spatial draw, spatial push and shared push for conveying the news nourish to its clients.

In light of an exact created cost display for every approach, Geo-Feed utilizes a savvy choice calculation that chooses about utilizing these methodologies in a way that: (a) minimizes the framework overhead to deliver the area mindful news sustain, and (b) ensures a specific reaction time for every client to get her area mindful news encourage. Geo-Feed additionally extends the spatial push way to deal with bolster the moving clients. Exploratory outcomes, in light of genuine and manufactured information, demonstrate that Geo-Feed is ideal over existing news encourage frameworks, with a negligible framework overhead.[1]

B) Mobi-Feed: A Location-Aware News Feed Framework for Moving Users: Mobi-Feed that is a system intended for planning area mindful news bolsters for portable clients. Mobi-Feed has three key capacities: area expectation, pertinence measure, and news bolster scheduler.

This proposed the n-look-ahead heuristic and ideal news sustain schedulers that work with the areas. this assessed other two capacities to create news encourages for a client at her current and $\mathrm{n}$ future anticipated the execution of Mobi-Feed through broad analyses utilizing a genuine guide, a genuine area mindful informal community information set and an engineered information set. The test comes about demonstrate that Mobi-Feed gives productive and astounding news encourages for versatile users.[2]

C) Area Service Based on News-encourage: The D-MobiFeed an area mindful news bolster structure considers the significance and assorted qualities of news sustains when planning news nourishes for moving client. It goes for expanding the aggregate importance of created newsfeed and fulfilling the differing qualities limitation.

The two key issues in D-Mobi-Feed, in particular, choice and streamlining issues. The choice issue is demonstrated as a greatest stream issue and empowers D-Mobi-Feed to choose whether it can satisfy the assorted qualities limitation for news encourage. Test comes about in view of a genuine informal community information set slithered from Foursquare and a genuine street arrange demonstrate that D-Mobi Feed can productively give area and differences mindful news bolsters while keeping up their high caliber as far as relevance.[3]

\section{EXISTING SYSTEM}

Existing LANF structures fundamentally send the hugest geo-named messages to their customers. Grievously, the genuine limitation of such a present approach is, to the point that, a news maintain may contain messages related to a comparable region (i.e., reason for interest) or a comparative class of ranges (e.g., sustenance, preoccupation or amusement). A news reinforce is an average value of existing LBSNs. It engages flexible customers to post geo-marked messages and get contiguous customer delivered messages as news empowers at whatever time, wherever. The expanding issues focus on recouping an individual once-over of things with a particular level of contrasting qualities.

In MobiFeed, a spatial cross section structure's used to record all geo-named messages. Given a customer user's query, a range question is issued to the framework record to recoup the geo-marked messages, which are not delivered by $u$, associated with a zone point, a spatial degree, or a setting region meeting the request area.

After a flexible customer issues a zone careful news maintain request to MobiFeed, MobiFeed calls the territory desire ability to return $\mathrm{n}$ future zones for $\mathrm{u}$. Its scheduler then finds a game plan of confident messages for each of $n+1$ zones and calls the hugeness distribute ability to channel all contender messages that don't have a place with any top-orders and choose the congruity of each exceptional candidate message to $u$. The scheduler finally returns news support for each zone with the ultimate objective that the total congruity score is expanded.

\section{PROPOSED SYSTEM}

A structure MobiFeed that is intended to plan news bolsters for versatile users. MobiFeed takes the impediments of cell phones and the client inclinations into record and timetables the most pertinent geo - labelled messages to portable users. Unfortunately, MobiFeed has a noteworthy restriction that exclusive considers the importance of messages to clients, so a news nourish contain messages identified with a similar class and subsequently it block clients to find new places and exercises. In ordinary web seek/a recommender framework, theme expansion is a key technique to enhance client fulfilment.

The information from the phone is traded as the commitment to zone careful news. Depends on the information the territory is foreseen and the congruity measure is discovered. The arrangement related geomarked message is used with the information that the message is named or not. 
IARJSET

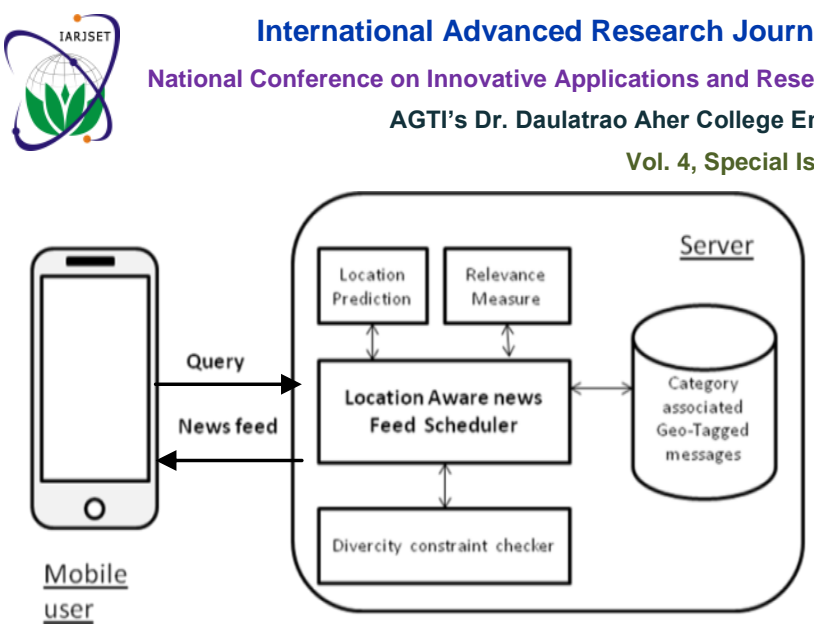

Fig2: Architecture

After this, the Diversity Constraint Checker is used to check the information status and it is transmitted to the mobile phone.

\section{a) Location Aware news support}

The range careful news sustain organizations, in two guideline regions. They are routine news support structures and range careful interpersonal associations. Standard News sustains structures. Most of existing news sustains systems work in like manner to distribute/subscribe organizations which use a push approach to manage fan out the message notice to every one of their customers. In any case, such structures are not significant to address the range careful news reinforce, as they don't consider the spatial congruity of each message, besides, the push approach does not scale up for broad number of distributers and supporters as it is the situation for casual associations. For business structures, our selective learning is about the Feeding Free for all system which we consider as our closest work and difference and it. The rule considered Bolstering Frenzy is to gather a cost show for picking subsequent to using the draw or push approaches as means for recuperating the news empower for an enrolled customer. The most ideal approach to use the Feeding Frenzy system for the region careful news urge issue is to associate a wrapper around it to channel any spatially insignificant message from the customers' news support.

In any case, that would be to a great degree inefficient as the spatial channel is associated as a thought by and large game plan. Our proposed system, MobiFeed, isolates itself from Feeding Craze in that it is worked with the area mindfulness usefulness as a principle need. As needs be, the question evaluation strategies, the cost model, and decision show consider the spatial part of each posted message close by the range of each customer. Existing business territory based casual associations fall in two classes, as plot. The primary class fuses that they consider the range information of the message underwriter as just an additional label annexed with the message. By then, a system customer will get a comparative news maintain paying little regard to the customer range. The second arrangement consolidates to having the region marks, they in like manner give their customers the ability to issue stretch out inquiries to see the whereabouts of their friends. MobiFeed perceives itself from all these business things in two essential perspectives:

(1) MobiFeed gives its customers the ability to set the spatial authenticity extent of each posted message., and from this time forward offer control to the message sponsor to pick who should get the posted information. For case, the atmosphere master association may pick a tornado forewarning is relevant to disciples discovered just in a particular district.

(2) Unlike each present system that are developed dominatingly to be used by phones, MobiFeed offers a more versatile way for the customers to share their geo labeled messages. Customers of MobiFeed can get to their record comparatively they use Facebook, notwithstanding, they will recuperate more relevant region careful news support than that of Facebook customers.

\section{b) Diversity Constraints}

The objective of contrasting qualities prerequisites is to give a methodical way to deal with considers the execution of these varying qualities constraints. To achieve this target, we first propose an authorized casing for these various qualities objectives, and exhibit to change these varying qualities goals into the acknowledged shape. With the acknowledged edge, these varying qualities impediments are as an upper-bound objective, and their specific confines all fall into a comparative range. On the most insignificant end of the extent of the limits, these different qualities necessities all utmost the conceivable locale of the portfolio progression issue to exactly one guide contrasting toward the also weighted regard. On the most bewildering end of the extent of the limits, these varying qualities objectives all get the opportunity to be unmistakably overabundance. By using a comparable regard for the upper bound of these contrasting qualities necessities in the standard shape, a proficiently relationship among them can be refined.

\section{A. Background of project:}

A mobi-feed framework is the accumulation of different voyaging related information, that's why it will be beneficial for any versatile client which give present and up and coming areas data. With the advances in situating systems, for example, GPS, cell-towers and Wi-Fi, clients can appreciate area based administrations more effectively than any time in recent memory, e.g., on their PDAs and GPS gadgets. Therefore, area based administrations have been exceptionally mainstream. The majority of the area based administrations can be dreamy as various sorts of spatial inquiries to a POI database or the street systems, for cases, 1) spatial range inquiry, which may locate the close-by eateries in a given spatial range from the clients, 2) k-closest neighbour question, which may discover the closest $\mathrm{k}$ service stations from the client's present area, 3) shortest way question, which can give the client with the most helpful course to her goal. In any case, with the ubiquity of proposal (e.g., Amazon \&Netflix) and 
IARJSET

socialization (like Facebook and Twitter) functionalities in the web benefits, the term Web 2.0 gets to be extremely famous, which is connected with web applications that encourage participatory data sharing, swarm sourcing, interoperability, client focused outline, and cooperation on the World Wide Web. The fame of Web 2.0 occurred as an immediate consequence of the wide increment of online client produced substance and long range informal communication innovations. In Web 2.0, the World Wide Web has moved from being an interface for data recovery to an intelligent medium where clients can share data, transfer client created content, and interface with different clients.

\section{B. Demand of Project:}

- Advantages:

1. Maximize the significance of the area based nourish while the bolster fulfilled the h-expanded requirement to differentiate the substance of the nourish.

2. To enhance the nature of the news nourish by not simply looking for the comparable substance but rather including all the client created redesigns from a differentiated arrangement of classifications.

- The application significantly utilizes 4 primary calculations to produce important yields and make a news nourish for the client:

1) Way Predicting- To decide the correct geo-area of the client in order to accumulate all the news overhauls from every one of the clients close-by and display it to the collector.

2) Classified Relevance Measuring- To furnish the client with various classifications to pick as his inclinations and create message which are important to that specific class.

3) News Feed producing- Create a news nourish with every one of the redesigns of different clients around that geo-area.

4) Enhancement checking- To create messages similarly from various picked classifications and making it broadened while being introduced to the client.

\section{CONCLUSION}

In this paper, we exhibited MobiFeed that is a system intended for booking area mindful news sustains for versatile clients. In this an area mindful MobiFeed considers the pertinence and differing qualities of news bolsters when planning news sustains for moving clients. MobiFeed clients can indicate the base number of classifications in a news sustain as an assorted qualities imperative, and it goes for expanding the aggregate importance of created news nourishes and fulfilling the differing qualities constraint. We concentrate on two key issues in MobiFeed, specifically, choice and streamlining issues. The choice issue is displayed as a most extreme stream issue and empowers MobiFeed to choose whether it can satisfy the differing qualities requirement for a news bolster. We portrayed the three key elements of MobiFeed, in particular, area forecast, pertinence measure, and news sustain scheduler. The area expectation capacity is intended to assess a client's area in view of the way forecast calculation. The pertinence measure capacity is actualized by incorporating non-spatial and spatial elements into the vector space model to gauge the significance of a message to a client.

We outlined a heuristic news encourage scheduler that works with the other two capacities to create news bolsters for a client at her current and look-ahead areas. Evaluated newsfeed and fulfilling the differing qualities imperative. Trial comes about, in view of genuine and engineered information; demonstrate that MobiFeed is good over existing news bolster frameworks, with a negligible framework overhead. It give ensures a specific reaction time for every client to acquire her area mindful MobiFeed And minimizes the framework overhead to deliver the area mindful news nourish. The MobiFeed gives effective and great news nourishes for portable clients.

\section{REFERENCES}

[1] Jie Bao1,Mohamed F,Mokbel1,Chi-YinChow2. "Geo-Feed: A Location-Aware News Feed System". Department of Computer Science and Engineering, University of Minnesota, Minneapolis, MN, USA.

[2] Wenjian Xu $\cdot$ Chi-Yin Chow $\cdot$ Man Lung ,Yiu $\cdot$ Qing Li $\cdot$ Chung Keung Poon . "Mobi-Feed: A Location-Aware News Feed Framework for Moving Users".May,2015,IEEE Transactions on services computing.

[3] K.Prathiba1, A.Petrisia2 1PG Student, Associate Professor Department of MCA, Dhanalakshmi Srinivasan College of Engineering and Technology. "Location Service Based on Newsfeed" International Journal of Research in Engineering Technology - Volume 1 Issue 4, May - June 2016.

[4] Siddharth Mishra,Toom Arjun Reddy,Pradeep Sadanand."A Location based diversified mobile news feed system(Android").March 2016,JETIR(ISSN-2349-5162)

[5] Jie Bao. "Towards Location-aware News Feeds and Recommendations". June 2014.

[6] J. Bao, M. F. Mokbel, and C.-Y. Chow." Geo-Feed: A locationaware news feed system". In IEEE ICDE, 2012.

[7] 2010 Census TIGER/Line Shapefiles. http://www.census. gov/geo/www/tiger/tgrshp2010/tgrshp2010.html.

[8] L. Aalto, N. G"othlin, J. Korhonen, and T. Ojala. "Bluetooth andWAP push based locationaware mobile advertising system." In ACM MobiSys, 2004.

[9] G. AdWords. http://adwords.google.com/.

[10] G. Aggarwal, G. Goel, C. Karande, and A. Mehta. "Online vertexweighted bipartite matching and single-bid budgeted allocations." In SIAM SODA, 2011.

[11] N. Armenatzoglou, S. Papadopoulos, and D. Papadias." A general framework for geo-social query processing”. In VLDB, 2013.

\section{BIOGRAPHIES}

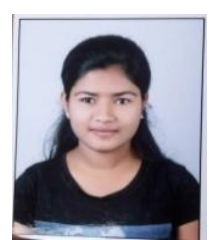

Pooja D. Kadam received Diploma in Computer Technology from S.V.P.M's College of engineering Malegaon (BK), Baramatiin 2014. Appearing B. E. degree at of Dr. Daulatrao Aher College of Engineering, Karad, District Satara. 
IARJSET

International Advanced Research Journal in Science, Engineering and Technology

National Conference on Innovative Applications and Research in Computer Science and Engineering (NCIARCSE-2017) AGTI's Dr. Daulatrao Aher College Engineering, Vidyanagar Extension, Karad

Vol. 4, Special Issue 4, January 2017

Archana T. Mulik received Diploma in Computer Science and Technology from Bharati Vidyapeeth Jawaharlal Nehru Institute of Technology Pune, Maharashtra in 2004. B. E. degree from Government College of Engg. Karad Maharashtra in Information Technology in 2011, the M. Tech degree from Bharati Vidyapeeth College of Engg. Pune, Maharashtra in 2014. Since July 2011 she is working as an Assistant Professor in Information Technology Department of Dr. Daulatrao Aher College of Engineering, Karad, District Satara, Maharashtra. Her area of interest includes Networking, Web Technology. 